\title{
Terminal restriction fragment patterns (TRFPs), a rapid, PCR-based method for the comparison of complex bacterial communities
}

\author{
Brian G. Clement, Lucia E. Kehl, Kristin L. DeBord, Christopher L. Kitts* \\ The Environmental Biotechnology Institute, Biological Sciences Department, California Polytechnic State University, San Luis Obispo, \\ CA 93407, USA
}

\begin{abstract}
Microbial populations in complex environmental samples are difficult to characterize; current techniques are incomplete and time consuming. We investigated a polymerase chain reaction (PCR)-based method for rapidly comparing bacterial communities independent of culture or cloning. Community 16S rRNA genes were amplified and fluorescently labeled by PCR. The labeled products were digested by a restriction enzyme and the labeled, terminal restriction fragments (TRFs) were separated by electrophoresis and detected by laser-induced fluorescence on an automated gene sequencer. PCR parameters were optimized using an in vitro model community of known organisms. Community comparisons were made between deer fecal pellets, petroleum hydrocarbon-contaminated sands and pristine sand. Principal components analysis (PCA) was used to compare the resulting TRF patterns (TRFPs). Patterns derived from a single enzyme digest did not result in accurate community characterizations. Accurate characterizations reflecting the expected bacterial community biology were only achieved by combining TRFP data derived from different enzyme digestions. Suggestions are offered for future use of this technique.
\end{abstract}

Keywords: 16S rRNA; Bacterial communities; PCR; Principal components analysis; Restriction analysis; TRFP

\section{Introduction}

Microbial ecology studies are limited by the complication of identifying and counting the microorganisms present in any given ecosystem. Visual observations are limited by the small number of possible morphologies. Biochemical tests, while more informative than morphological studies, are restricted to cultured species. Genetic techniques, particularly those that utilize the polymerase chain reaction (PCR) to amplify sample DNA, are more useful than culture or direct observation for assessing sample diversity [1-5]. While PCR-based methods can assess a wider range of organisms than culture techniques, they are limited by inherent biases. Sequence-based differential amplification and/or poor homology between templates and 'consensus' primers can result in unequal or no amplification of certain templates in a community [6,7].

To date, most PCR-based methods rely on amplifying, cloning and sequencing 16S rRNA genes (16S rDNA) from sample DNA. Cloning can detect both known and unknown sequences in a sample, but the process is time consuming. One must sequence several thousand clones from a single sample to 
definitively characterize a soil community that may harbor as many as 4000 species per gram [8]. Identification of individual community members, however, is not required for substantive studies of microbial communities.

Patterns derived from the diversity of $16 \mathrm{~S}$ rDNA sequences in a microbial community have been used to rapidly characterize environmental samples $[2,3]$. One example, amplified ribosomal DNA restriction analysis (ARDRA), creates patterns by digesting amplified community 16S rDNA with restriction enzymes $[9,10]$. While faster than cloning, ARDRA data relies on the visual resolution of all restriction fragments. Visual detection may not resolve a significant fraction of the fragments, thereby reducing the amount of data available for analysis. Additionally, the number of restriction fragments per organism presents a problem when community restriction fragments are visually resolved. Restriction fragment analysis effectively divides the visual signal of any one organism's DNA. Therefore, fragments from organisms with a large number of restriction sites may be eliminated from the resulting pattern while fragments from those organisms with few restriction sites are more likely to appear in the pattern. Ideally, a community restriction pattern technique would both resolve all available fragments and limit those fragments to one per organism.

Fluorescent-labeled PCR, in which one or both primers carry a $5^{\prime}$ fluorescent molecule, can be followed by restriction enzyme digestion to produce fluorescent-labeled terminal restriction fragments. This technique was previously evaluated with in vitro cultures as a means of identifying individual bacteria [11,12], and its utility for complex samples was modeled using a sequence database [13]. Patterns reflecting genetic diversity are produced by electrophoretic separation and detection of only the labeled terminal restriction fragments (TRFs) on an automated gene sequencer. In these patterns, each TRF can be used as a data point for community comparison via statistical analyses.

Using 16S rDNA sequences for differentiating bacteria based on TRF length cannot produce the ideal of one data point per species. A TRF pattern (TRFP) produced from amplified 16S rDNA is a simplified representation of the community. Different species, regardless of taxon, may produce identical length TRFs with a given restriction enzyme [13]. However, organisms that produce identical TRFs with one restriction enzyme may produce unique TRFs with a different restriction enzyme. Thus, a more accurate community representation may be achieved by combining multiple TRFPs derived from different restriction enzyme digests.

Here we report the use of TRFPs in the analysis of different microbial communities; in vitro communities, deer fecal pellets, and coastal dune sands. In vitro control communities were used to optimize methodology, while deer fecal pellets were examined as an example of complex sample communities where inter-sample similarity was expected. Pristine sands and petroleum hydrocarbon-contaminated sands were examined to investigate changes in TRFPs that might correspond to a defined environmental change.

\section{Materials and methods}

\subsection{Organisms and samples}

In vitro communities were created by combining DNAs extracted from a pure culture of each strain listed in Table 1. Deer (Odocoileus hemionus) fecal pellets were collected in sterile tubes on the campus of California Polytechnic State University, San Luis Obispo, and used for DNA isolations within $24 \mathrm{~h}$. Pellets from two discrete piles were used and labeled Deer 1 and Deer 2. Pristine (Sand 1) and petroleum hydrocarbon-contaminated sand were collected from a coastal oil field at Guadalupe, CA, and stored at $4^{\circ} \mathrm{C}$ until used. The petroleum contaminants were either a mid-distillation cut (Sand 2), approximately $\mathrm{C}_{8}$ to $\mathrm{C}_{36}$, or crude and waste oil (Sand 3), approximately $\mathrm{C}_{8}$ to $\mathrm{C}_{42}[14]$.

\subsection{DNA isolations}

Both in vitro culture and sand DNA were isolated by a phenol:chloroform-isoamyl alcohol method modified from Sambrook et al. [15] by the addition of a lysis step. Cell lysis was accomplished by incubating $(24 \mathrm{~h}$ for sand and $1 \mathrm{~h}$ for cultures at $\left.28^{\circ} \mathrm{C}\right) 200 \mu \mathrm{l}$ of pelleted cells in $200 \mu \mathrm{l}$ of lysis 
buffer consisting of $100 \mathrm{mM} \mathrm{NaCl}, 10 \mathrm{mM}$ Tris $\mathrm{HCl}$, $25 \mathrm{mM}$ disodium EDTA and $700 \mathrm{U} / 1$ lysozyme.

DNA from deer fecal pellets was isolated using the FastDNA Medium-Hard Tissue kit with a FastPrep bead beater (all kit components from Bio 101 , Vista, CA, USA). Prior to processing, $50 \mathrm{mg}$ of fecal pellet was incubated in $250 \mu \mathrm{l}$ MT Buffer (Bio 101) and $750 \mu \mathrm{l} 200 \mathrm{mM}$ sodium phosphate buffer ( $\mathrm{pH}$ 8.0) for $24 \mathrm{~h}$ at $4^{\circ} \mathrm{C}$ in MH FastDNA tubes (Bio 101). The tubes were processed on the bead beater for $5.0 \mathrm{~s}$ at $5.0 \mathrm{~m} / \mathrm{s}$. The incubation and processing were repeated. DNA was then isolated according to the manufacturer's SpinFilter protocol (Bio 101).

DNA isolated from all sample types was visualized on a $1.5 \%$ agarose gel in $\mathrm{pH} 8.25$ TBE buffer (89.2 $\mathrm{mM}$ Tris base, $88.9 \mathrm{mM}$ boric acid, $2.47 \mathrm{mM}$ disodium EDTA) stained with ethidium bromide and quantified by comparison with DNA standards (MspI digest of pUC18, Sigma, St. Louis, MO, USA).

\section{3. $P C R$}

Primers homologous to $16 \mathrm{~S}$ rDNA conserved regions were used to amplify a fragment from bases 8-536 in E. coli. This region and the primer binding sites were identified as optimal for this application using the author's spreadsheet macro analysis of published 16S rRNA sequences [16]. Primers were adapted from Bruce et al. [17] by the addition of a $\mathrm{G}$ to the $3^{\prime}$ end of the reverse primer, K2R. The forward primer, $\mathrm{K} 1 \mathrm{~F}$, was $5^{\prime}$ labeled with $\mathrm{FAM} \AA$, a fluorescent sequencing dye (Perkin-Elmer Applied Biosystems Division, Foster City, CA, USA). The sequences of $\mathrm{K} 1 \mathrm{~F}$ and $\mathrm{K} 2 \mathrm{R}$ were as follows: $\mathrm{K} 1 \mathrm{~F}$, 5'-AGA GTT TGT TCE TGG CTC AG-3' (consensus for eubacterial SSU rDNA [17]); K2R, 5'-GTA TTA CCG CGG CTG CTG G-3' (universal to all SSU rDNA [17]). The underlined $\mathrm{T}$ in K1F replaces an $\mathrm{A}$ from the consensus primer to eliminate a Sau3A cut site. The underlined $\mathrm{C}$ is an additional one-base pair mismatch with $\mathrm{K} 1 \mathrm{~F}$ for the published $P$. vulgaris $16 \mathrm{~S}$ rDNA sequence [16]. All other in vitro community organisms used had one or fewer mismatches.

Reactions were carried out in $50 \mu$ with $1 \times$ buffer (Perkin-Elmer), $0.6 \mathrm{mM}$ dNTP, $8 \mu \mathrm{g} / \mathrm{ml}$ bovine serum albumin, $2 \mathrm{mM} \mathrm{MgCl}{ }_{2}$, and $1 \mathrm{U}$ of Taq DNA polymerase (Perkin-Elmer). To overcome inhibitors, template DNA for fecal and sand samples was diluted to $0.5 \mathrm{ng}$ per reaction, at which point all samples amplified equally. Two in vitro communities were constructed by combining either equal or random amounts of DNA extracted from pure cultures. Reaction temperatures and cycling for fecal and sand samples were: $94^{\circ} \mathrm{C}$ for $2 \mathrm{~min}, 35$ cycles of $94^{\circ} \mathrm{C}$ for $1 \mathrm{~min}, 48.5^{\circ} \mathrm{C}$ for $1 \mathrm{~min}, 72^{\circ} \mathrm{C}$ for $2 \mathrm{~min}$, followed by $72^{\circ} \mathrm{C}$ for $10 \mathrm{~min}$. In vitro communities were subjected to the same PCR parameters with the addition of successive trials at annealing temperatures of 55,51 and $45^{\circ} \mathrm{C}$. Triplicate reactions were prepared for sand and fecal samples. Products were visualized on $2.0 \%$ agarose gels and quantified as above.

\subsection{Primer removal and amplicon concentration}

For sand and fecal pellet samples, replicate PCR reactions were combined in $30-\mathrm{kDa}$ molecular weight cut-off filters and concentrated. The concentrate was washed twice and resuspended in sterile deionized water. Resuspension volumes were varied to equalize amplicon concentrations.

\subsection{Amplicon digestion}

Digestion reactions contained $0.1 \mu \mathrm{g}$ of labeled DNA and were incubated for $12 \mathrm{~h}$ at $37^{\circ} \mathrm{C}$. The $15-\mu$ reactions contained $1.3 \mathrm{U} / 1$ of either Sau3A, HaeIII, MspI (New England Biolabs, Beverly, MA, USA) or HhaI (Promega, Madison, WI, USA) in the manufacturer's recommended reaction buffers.

\subsection{Electrophoresis and fragment size determination}

Electrophoresis samples containing $2 \mu \mathrm{l}$ of digestion reaction, $5 \mu \mathrm{l}$ formamide (BioRad, Benecia, CA, USA), $1 \mu$ l load buffer and $1 \mu$ ROX500® size standard (Perkin-Elmer, Applied Biosystems Inc., Fremont, CA, USA) were denatured at $95^{\circ} \mathrm{C}$ for 5 min. Approximately $2 \mu \mathrm{l}$ of each sample was applied to a $6 \%$ polyacrylamide (19:1 bis from BioRad) gel and run at $30 \mathrm{~W}$ for $16 \mathrm{~h}$ on an ABI 373 Sequencer. TRF sizing was performed with the electropherogram output from GeneScan 2.0.2 software using a third-order least-squares size curve and heavy smoothing. Manual peak calling was made for peaks 
with a minimum height of 20 fluorescence units above background. Fragments were resolved to one base pair by manual alignment of the size standard peaks from different electropherograms. Inconsistencies in the Genescan software peak calling routine prevented the use of peak area data.

\subsection{Data analysis}

TRFPs from all five environmental samples were compared using principal components analysis. Sample data consisted of Boolean character sets ( 1 or 0 ) corresponding to the absence or presence of a given TRF in a pattern. The total number of elements analyzed equaled the number of unique TRFs observed in all samples digested with the same enzyme. Analyses were performed for each set of samples digested with a given enzyme and for all three enzyme digestions combined.

\section{Results and discussion}

\subsection{Single organism terminal restriction fragments}

In order to identify individual organisms in a TRFP from the in vitro community, TRF lengths were determined for all organisms used. In cases where database entries existed for the organism, TRF lengths were within $5 \mathrm{bp}$ of the predicted length. An extra TRF was observed for some organisms. In most cases the shortest TRF was the most abundant fragment. Longer TRFs corresponded to either undigested amplicon or the predicted length of partial digestion products. M. luteus, P. fluorescens, A. tumefaciens and $M$. phlei consistently produced multiple TRFs, possibly indicating 16S rDNA sequence heterogeneity in these organisms (Table 1). Multiple heterogeneous $16 \mathrm{~S}$ rDNAs in a single organism have been previously observed in Mycobacterium terrae [18] and some marine isolates [19]. Digestion with MspI resulted in similar or identical length TRFs for five different organisms (Table 1). Therefore, MspI was excluded from further testing with the in vitro community. Sau3A and HhaI resulted in unique TRF lengths for all organisms, although some TRFs differed in length by a single base pair (Table 1). Hae III was not tested on the in vitro communities.

\subsection{In vitro community TRFPs}

The creation of TRFPs from the in vitro community was used to optimize PCR and enzyme digestion reactions. When the PCR annealing step was $48.5^{\circ} \mathrm{C}$, expected TRFs corresponding to all in vitro com-

Table 1

Predicted and observed TRF lengths for in vitro community organisms

\begin{tabular}{|c|c|c|c|c|c|c|c|}
\hline \multirow[t]{2}{*}{ Organism } & \multirow{2}{*}{$\begin{array}{l}\text { Amplified } \\
\text { fragment } \\
\text { length: } \\
\text { predicted }\end{array}$} & \multicolumn{2}{|c|}{$\begin{array}{l}\text { TRF length after } \\
\text { digestion with Sau3A: }\end{array}$} & \multicolumn{2}{|c|}{$\begin{array}{l}\text { TRF length after } \\
\text { digestion with } M s p \mathrm{I} \text { : }\end{array}$} & \multicolumn{2}{|c|}{$\begin{array}{l}\text { TRF length after } \\
\text { digestion with } H h a \mathrm{I} \text { : }\end{array}$} \\
\hline & & predicted & observed & predicted & observed & predicted & observed \\
\hline Proteus vulgaris & 529 & 121 & $\mathbf{1 2 5}, 278$ & 496 & 497 & 213 & 214 \\
\hline Mycobacterium phlei & 503 & 113 & $118, \sim 500$ & 160 & $163,284^{\mathrm{a}}$ & 367 & 368 \\
\hline Enterobacter aerogenes & NDE & NDE & 278, $\sim 520$ & NDE & 497 & NDE & 371 \\
\hline Micrococcus luteus & 505 & 505 & $215^{\mathrm{a}}, \sim 500$ & 163 & 165 & 369 & $\sim 510, \sim 515^{\mathrm{a}}$ \\
\hline Citrobacter freundii & 529 & 273 & $\mathbf{2 7 7}, \sim 520$ & 496 & 494, $\sim 525$ & 373 & 373 \\
\hline Psuedomonas fluorescens & NDE & NDE & $\mathbf{1 9 4}, 271$ & NDE & 493 & NDE & $155, \sim 530$ \\
\hline Streptococcus faecalis & NDE & NDE & 323, 437 & NDE & $\sim 545$ & NDE & 218 \\
\hline Alcaligenes faecalis & 529 & 196 & 200, 277 & 496 & 497 & 67 & 67 \\
\hline Agrobacterium tumefaciens & 471 & 240 & 244, 471 & 402 & $128^{\mathrm{a}}, 407$ & 340 & 339, 470 \\
\hline Staphylococcus hominis & 537 & 305 & $\mathbf{3 1 0}, 426$ & 156 & 156, $\sim 540$ & 239 & 239 \\
\hline Flavobacterium indolgenes & 518 & 66 & 69 & 202 & $\mathbf{2 0 6}, 488$ & 225 & 227 \\
\hline
\end{tabular}

NDE, no database entry for predictions [16]. Bold entries indicate a TRF whose peak area was greater than $40 \%$ of total peak areas. Note: electropherogram peaks resulting from fragments longer than 500 base pairs were resolved to $\pm 5 \mathrm{bp}$.

${ }^{a}$ Peak of unknown origin; not a predicted partial digestion product. 
munity organisms except $P$. vulgaris were detected. Annealing temperatures greater than $49^{\circ} \mathrm{C}$ resulted in the loss of some fragments while temperatures less than $48.5^{\circ} \mathrm{C}$ did not increase fragment recovery (Fig. $1)$. TRFPs for the in vitro community were identical among replicate digestions for all enzymes tested (data not shown). Although some organisms in the community produced secondary TRFs (Table 1), the community TRFPs were reproducible and consistent (data not shown).

\subsection{Bacterial community TRFPs from environmental samples}

The optimum PCR parameters identified using the in vitro community were next applied to create TRFPs from environmental samples. The choice of

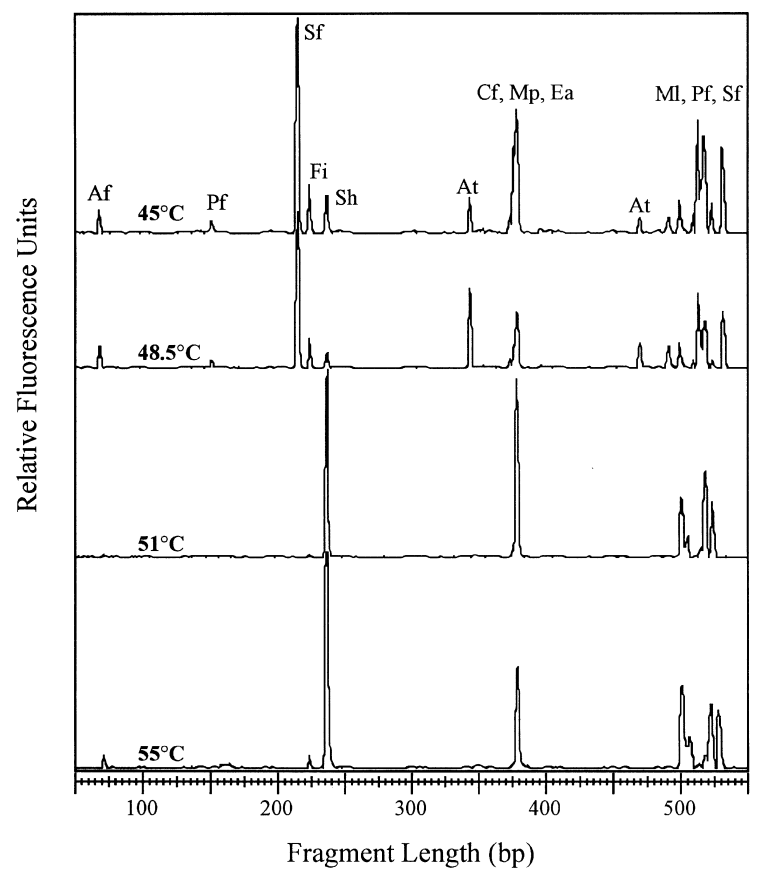

Fig. 1. Electropherograms of the in vitro community subjected to five different annealing temperatures during PCR and digested with HhaI. Labeled peaks correspond to the TRF for individual organisms (Table 1). Abbreviations of organisms: Af, Alcaligenes faecalis; At, Agrobacterium tumefaciens; Cf, Citrobacter freundii; Ea, Enterobacter aerogenes; Fi, Flavobacterium indolgenes; Ml, Micrococcus luteus; Mp, Mycobacterium phlei; Pf, Psuedomonas fluorescens; Sf, Streptococcus faecalis; Sh, Staphylococcus hominis.
Table 2

Number of terminal restriction fragments per pattern

\begin{tabular}{lllll}
\hline Sample & MspI & Hae III & HhaI & $\begin{array}{l}\text { Total number } \\
\text { of TRFs }\end{array}$ \\
\hline Deer 1 & 24 & 35 & 36 & 95 \\
Deer 2 & 26 & 48 & 32 & 106 \\
Sand 1 & 56 & 60 & 36 & 152 \\
Sand 2 & 30 & 50 & 39 & 119 \\
Sand 3 & 41 & 37 & 30 & 108 \\
\hline
\end{tabular}

enzyme greatly affected the number of TRFs visible in any one pattern. The enzyme HaeIII gave the greatest number of TRFs in three out of five samples. The maximum number of distinguishable TRFs as postulated by Brunk et al. [13] was not reached with any of these samples. Each TRFP had 25-60 discernible TRFs that could be used to differentiate one bacterial community from another (Table 2). Fig. 2 shows complete HaeIII TRFPs as an example; Fig. 3 shows the one-base pair resolution of the technique. Visual inspection of the TRFPs in Fig. 2 provides intuitive confirmation of the differences between the bacterial communities sampled. The deer samples appear to be very similar and still quite different from all the sand samples. However, an objective analysis of community relatedness can be achieved

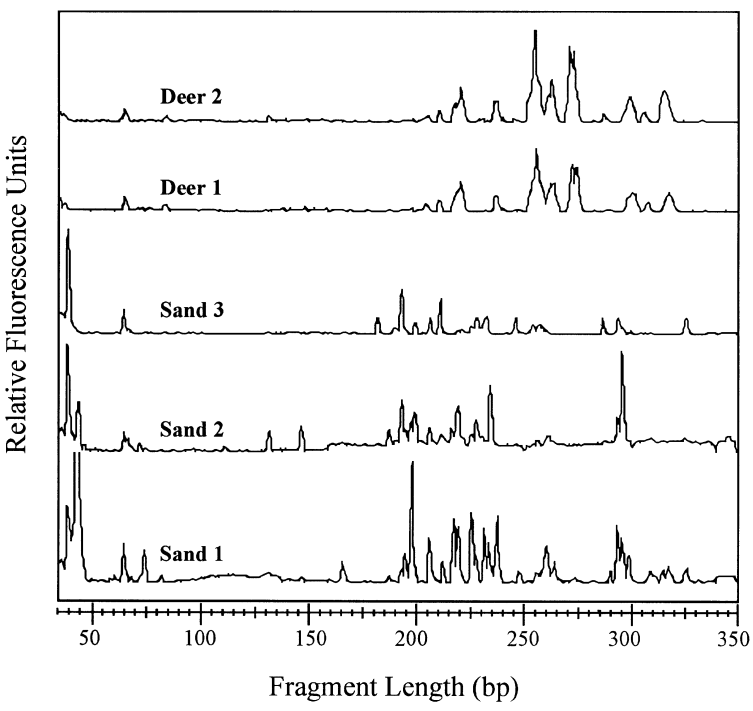

Fig. 2. Electropherograms of the TRFPs derived from HaeIII digestions of environmental bacterial community DNAs. 


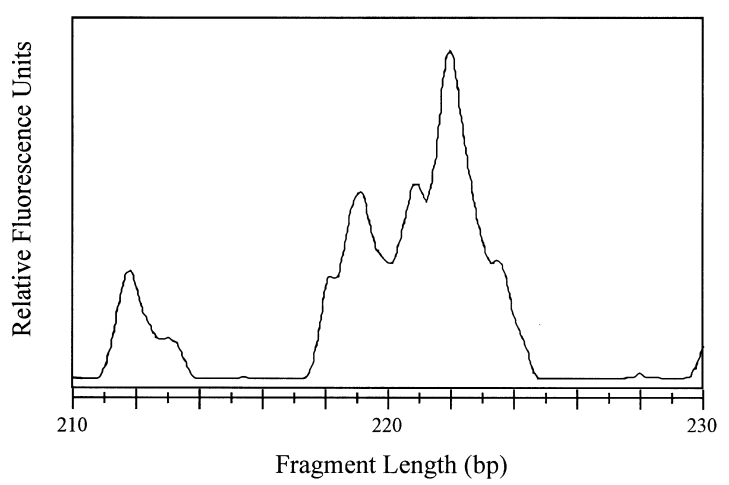

Fig. 3. Close-up of the HaeIII TRFP from Deer 2 showing $1 \mathrm{bp}$ TRF length resolution.

only by assigning a defined length to each TRF in a pattern.

Several issues are important when calling peaks to one-base pair resolution. We found Genescan version 2.0.2 software inconsistent in its recognition of peaks. However, manual peak calling introduces a subjective element to data collection. To limit this effect we maintained a dual panel view with samples in one panel and the internal size standards in the second. This allowed alignment of two different electropherograms and the comparison of sample peaks to standard peaks. Even with these procedures, wide peaks and those with 'shoulders' presented problems. Size standard peaks tend to widen and develop shoulders with an increase in fragment size. Therefore, resolution is lost with increasing fragment sizes. We found that consistent well-formed peaks were rare for TRFs greater than 350 base pairs, and therefore our analysis only included peaks smaller than 351 base pairs.

\subsection{Multivariate analysis of TRFPs}

Principal components analysis (PCA) of the TRFPs arranged the five samples differently depending on the enzyme used to create the pattern (Fig. $4 a-c)$. Analysis of the MspI and HaeIII TRFPs alone failed to produce significant clusters. The HhaI TRFPs did place the deer fecal samples together, but also paired a petroleum hydrocarbon-contaminated sand (Sand 2) with the pristine sand (Sand 1). However, when the information from all three digests was combined, each community TRFP had
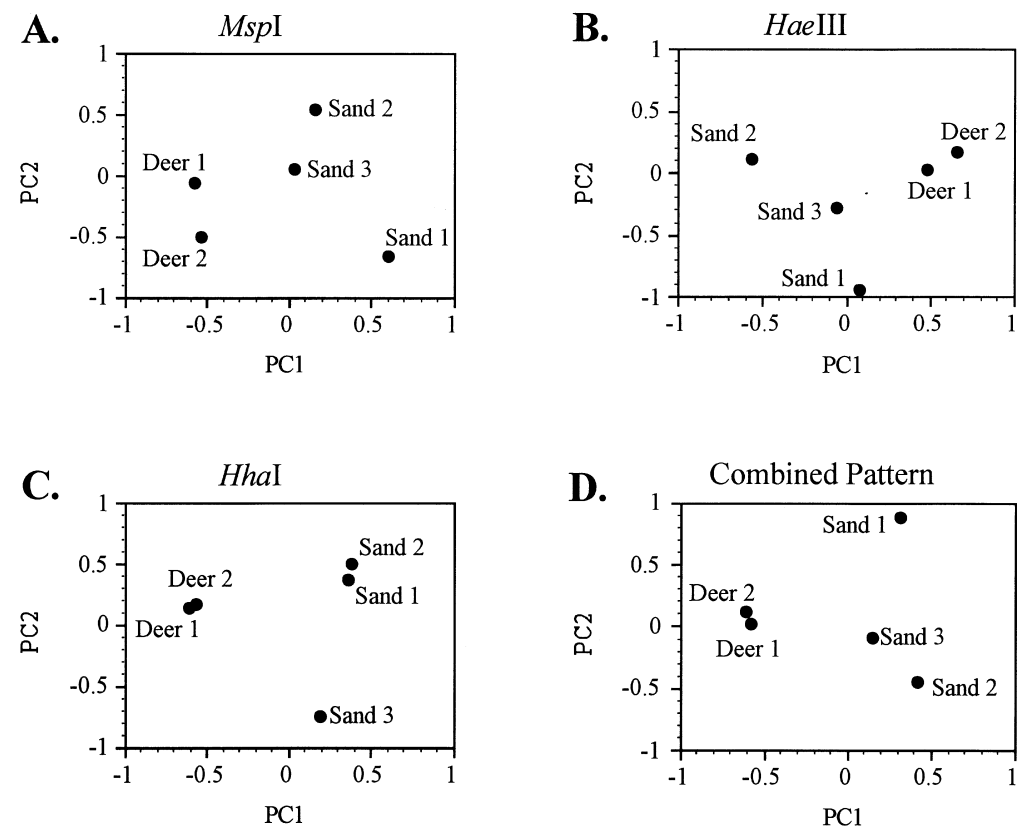

Fig. 4. Principal component analyses of TRFP data sets from environmental samples. Data sets derived from digestions with: (A) MspI; (B) Hae III; (C) HhaI; and (D) all three digests combined. 
from 98 to 152 elements for analysis (Table 2). PCA of the combined data resulted in a clear pairing of the two deer fecal samples (Fig. 4d). Principal component 1 (PC1) separated deer fecal samples from sand samples, and principal component 2 (PC2) separated the pristine sand from the two petroleumcontaminated sands. Thus the PCA results correlated with the expected bacterial community biology of each sample best when the data from multiple enzyme digestions was combined. Essentially, the complex pattern expected from the community is only reflected in the combination of several relatively simple TRFPs.

\subsection{Limitations of the method}

Our work has identified some limitations of the TRFP technique as described here. (i) The tendency of PCR to differentially amplify templates prevented quantification of relative abundance in the community [6]. The in vitro community DNA produced TRFs of unequal relative abundance (electropherogram peak area) with different annealing temperatures (Fig. 1). Thus, TRF abundance does not reflect an organism's abundance within the sample community. Furthermore, differential amplification can result in the 'loss' of some community templates. For example, the TRF corresponding to $P$. vulgaris was not observed in the in vitro TRFP (Fig. 1, Table 1). (ii) Individual TRF detection was limited by electrophoresis technology. One-base pair resolution up to $600 \mathrm{bp}$ would allow maximum information retrieval from PCR with the chosen primer set. Such resolution may be achieved by the new generation of automated capillary gel electrophoresis DNA sequencers. (iii) As expected, patterns derived from a single enzyme digest did not result in accurate community characterizations (Fig. 4). Accurate characterizations were only achieved by combining TRFP data derived from different enzyme digestions. (iv) Statistical analysis for community comparison was restricted to Boolean ( 1 or 0 ) data due to software shortcomings. Peak area could be included in TRFP analysis with the use of more capable integration software. We are investigating GelCompar® (Applied Math, Belgium) software for this purpose.

\section{Conclusions and recommendations}

Distinctive bacterial community patterns can be rapidly generated using fluorescently labeled, PCRamplified 16S rDNA terminal restriction fragments using the method described here (TRFP). TRFPs will be useful for investigating complex communities where community dynamics are on a time scale that requires rapid analysis and the number of bacterial species in a sample may be quite large. Composed of only the terminal restriction fragment(s) from each organism, TRFPs represent a manageable data set. Although different organisms, even unrelated species, produced the same TRF with a given restriction enzyme digestion (Table 1, [13]), this information reduction was mitigated by the use of multiple enzymes (Fig. 4). Organisms that produce identical length TRFs with one restriction enzyme may produce different length TRFs when digested with another restriction enzyme (Table 1). Thus, the best community comparisons result from analysis of the combined data from different enzyme digests (Fig. 4).

This paper does not address our work-in-progress on the effects of DNA extraction, template concentration, and PCR bias on the reproducibility of TRFPs from environmental samples. However, the similarity in TRFPs from different deer fecal pellets (Fig. 2) indicates a high level of reproducibility may be possible. At present, to best describe a bacterial community, we make the following conservative recommendations: first, combine multiple DNA extractions from each well-homogenized sample to minimize extraction bias. Similarly, combine amplicons from multiple PCRs to reduce PCR bias. Load as much as DNA from each digestion as is practical onto the polyacrylamide gel to allow detection of less abundant fragments. Finally, combine TRFP data from three or more different restriction enzyme digests to increase the information derived from each community.

\section{Notation}

A adenine

ABI Applied Biosystems Incorporated (Per- 


\section{kin-Elmer Applied Biosystems Divi- sion)}

ARDRA amplified ribosomal DNA restriction analysis

C cytosine

DNA deoxyribonucleic acid

G guanine

PCA principal components analysis

PCR polymerase chain reaction

rDNA ribosomal DNA

rRNA ribosomal ribonucleic acid

$\mathrm{T}$ thymine

TRF terminal restriction fragment

TRFP terminal restriction fragment pattern

\section{Acknowledgements}

None of this work would have been possible without the support and input of the director of the Environmental Biotechnology Institute, Dr. Raul Cano. This work was financially supported by a grant from the Unocal Corporation and a Cal Poly State Faculty Support Grant. The authors would like to thank Gonzalo Garcia for his advocacy and support.

\section{References}

[1] P. Hugenholtz, N.R. Pace, Identifying microbial diversity in the natural environment: a molecular phylogenetic approach, TIBTech 14 (1996) 190-197.

[2] M.T. Suzuki, M.S. Rappe, Z.W. Haimberger, H. Windfield, N. Adair, J. Strobel, S.J. Giovannoni, Bacterial diversity among small-subunit rRNA gene clones and cellular isolates from the same seawater sample, Appl. Environ. Microbiol. 63 (1997) 983-989.

[3] D.M. Ward, R. Weller, M.M. Bateson, 16S rRNA sequences reveal numerous uncultured microorganisms in a natural community, Nature 344 (1990) 63-65.

[4] R.-F. Wang, C. Wei-Wen, C.E. Cerniglia, PCR detection and quantitation of predominant anaerobic bacteria in human and animal fecal samples, Appl. Environ. Microbiol. 62 (1996) $1242-1247$.

[5] S. Weidner, W. Arnold, A. Puhler, Diversity of uncultured microorganisms associated with the seagrass halophila stipulacea estimated by restriction fragment length polymorphism analysis of PCR-amplified 16S rRNA genes, Appl. Environ. Microbiol. 62 (1996) 766-771.
[6] M.T. Suzuki, S.J. Giovannoni, Bias caused by template annealing in the amplification of mixtures of $16 \mathrm{~S}$ rRNA genes by PCR, Appl. Environ. Microbiol. 62 (1996) 625630.

[7] A.-L. Reysenbach, L.J. Giver, G.S. Wickham, N.R. Pace, Differential amplification of rRNA genes by polymerase chain reaction, Appl. Environ. Microbiol. 58 (1992) 34173418.

[8] J. Borneman, P.W. Skroch, K.M. O'Sullivan, J.A. Palus, N.G. Rumjanek, J.L. Jansen, J. Nienhuis, E.P. Triplett, Molecular microbial diversity of an agricultural soil in Wisconsin, Appl. Environ. Microbiol. 62 (1996) 1935-1943.

[9] M.R. Fries, G.D. Hopkins, P.L. McCarty, L.J. Forney, J.M. Tiedje, Microbial succession during a field evaluation of phenol and toluene as the primary substrates for trichloroethene cometabolism, Appl. Environ. Microbiol. 63 (1997) $1515-1522$.

[10] A. Massol-Deya, R. Weller, L. Rios-Hernandez, J.-Z. Zhou, R.F. Hickey, J.M. Tiedje, Succession and convergence of biofilm communities in fixed-film reactors treating aromatic hydrocarbons in groundwater, Appl. Environ. Microbiol. 63 (1997) 270-276.

[11] E. Avaniss-Aghajani, K. Jones, D. Chapman, C. Brunk, A molecular technique for identification of bacteria using small subunit ribosomal RNA sequences, BioTechniques 17 (1994) 144-149.

[12] E. Avaniss-Aghajani, K. Jones, A. Holtzman, T. Aronson, N. Glover, M. Boian, S. Froman, C.F. Brunk, Molecular technique for rapid identification of mycobacteria, J. Clin. Microbiol. 34 (1996) 98-102.

[13] C.F. Brunk, E. Avaniss-Aghajani, C.A. Brunk, A computer analysis of primer and probe hybridization potential with bacterial small-subunit rRNA sequences, Appl. Environ. Microbiol. 62 (1996) 872-879.

[14] Final Draft Feasability Study to Address Separate-Phase and Dissolved-Phase Diluent at the Guadalupe Oil Field, San Luis Obispo County, CA. Levine-Fricke Company, Santa Maria, CA. March 18, 1996.

[15] J.E. Sambrook, F. Fritsch, T. Maniatis, Molecular Cloning, vol. 3, Cold Spring Harbor Laboratory, Cold Spring Harbor NY, 1989, pp. E.3-4.

[16] B.L. Maidak, G.J. Olsen, N. Larsen, R. Overbeek, M.J. McCaughey, C.R. Woese, The RDP (Ribosmal Database Project), Nucleic Acids Res. 25 (1997) 109-111.

[17] K.D. Bruce, W.D. Hiorns, J.L. Hobman, A.M. Osborn, P. Strike, D.A. Ritchie, Amplification of DNA from native populations of soil bacteria by using the polymerase chain reaction, Appl. Environ. Microbiol. 58 (1992) 3413-3416.

[18] B. Ninet, M. Monod, S. Emler, J. Pawlowski, C. Metral, P. Rohner, R. Auckenthaler, B. Hirschel, Two different 16S rRNA genes in a mycobacterial strain, J. Clin. Microbiol. 34 (1996) 2531-2536.

[19] L. Kerkhof, M. Voytek, Multiple heterogeneous 16S rRNA genes in individual species of marine bacterial isolates, in: 97th General Meeting Abstracts, American Society for Microbiology (ASM), Washington DC, 1997. 